\title{
Medical termination of a partial hydatidiform mole and coexisting fetus during the second trimester: A case report
}

\author{
YINFENG WANG $^{1}$, HONGLANG QIAN ${ }^{1}$ and JINHUA WANG $^{2}$ \\ Departments of ${ }^{1}$ Gynecology and ${ }^{2}$ Radiology, Women's Hospital, Zhejiang University \\ School of Medicine, Hangzhou, Zhejiang 310006, P.R. China \\ Received January 11, 2015; Accepted September 4, 2015
}

DOI: $10.3892 / \mathrm{ol} .2015 .3743$

\begin{abstract}
Partial hydatidiform mole and coexisting fetus (PHMCF) is a rare condition that presents a dilemma for physicians and the parents of the fetus, particularly when PHMCF is detected during the second trimester of pregnancy. The present study reports a case of PHMCF terminated by induction of labor via administration of Rivanol at 17 weeks. Follow-up measurements of serum $\beta$-human chorionic gonadotropin $(\beta-\mathrm{HCG})$ levels, as well as imaging studies, indicated the presence of persistent trophoblastic disease (PTD) and lung metastases. The patient was therefore admitted for three courses of chemotherapy. Subsequently, the metastases receded and $\beta$-HCG levels decreased to within the normal range. The patient demonstrated no disease recurrence for 1 year. Following a review of the relevant literature, to the best of our knowledge, all PHMCF cases terminated by medical induction of labor during the second trimester resulted in the development of PTD and lung metastases. However, three cases of PHMCF that were terminated by caesarean section during the third trimester did not develop PTD or metastases. The present study therefore hypothesized that medical termination may not be a safe therapeutic strategy for the treatment of PHMCF during the second trimester, and that pregnancy should be allowed to continue empirically.
\end{abstract}

\section{Introduction}

Hydatidiform mole with a coexistent fetus (HMCF) is a rare condition, with an incidence of 1/22,000-1,000,000 pregnancies (1). There have been a number of cases of HMCF previously reported (2-4). The majority are complete HMCF cases, which possess a significant risk (19.5-62.5\%) of inducing persistent trophoblastic disease (PTD) (5). By contrast, persistent or

Correspondence to: Dr Jinhua Wang, Department of Radiology, Women's Hospital, Zhejiang University School of Medicine, 1 Xueshi Road, Hangzhou, Zhejiang 310006, P.R. China

E-mail: wangjinhua80119@163.com

Key words: partial hydatidiform mole, coexisting fetus, second trimester, medical termination, persistent trophoblastic disease, metastases metastatic trophoblastic disease following a partial mole is rare, with an estimated risk of $\sim 1-5.6 \%$ (6). However, to the best of our knowledge, there have been no previous reports investigating the risk of persistent or metastatic trophoblastic disease following partial HMCF (PHMCF), due to the rarity of this condition. The management of such pregnancies creates a dilemma for the physician and parents, particularly when PHMCF occurs during the second trimester of pregnancy. Medical termination is effective during the second trimester; however, this technique increases the risk of the occurrence of PTD when used to terminate molar pregnancies (7). The aim of this study was to investigate the safety of medical termination for patients with PHMCF. The present study reports a case of PHMCF in which the pregnancy was terminated and delivery was induced via intra-amniotic administration of Rivanol, followed by aspiration curettage, which resulted in the occurrence of PTD and pulmonary metastases. Written informed consent was obtained from the patient.

\section{Case report}

In November 2013, a 22-year-old female (gravida 2, para 0) was referred to the Department of Gynecology at the Women's Hospital of Zhejiang University School of Medicine (Zhejiang, China) at 16 weeks of gestation, with suspected molar pregnancy. Sonography and magnetic resonance imaging (MRI) revealed a fetus and placenta, which appeared normal, in the posterior portion of the uterus. However, an additional cystic placenta with a degenerated twin was identified in the right anterior portion of the uterus (Fig. 1). In addition, the patient's serum $\beta$-human chorionic gonadotropin $(\beta-\mathrm{HCG})$ levels were markedly increased (449,078 IU/1). The couple decided to terminate the pregnancy following consideration of the risks. Delivery was induced via intra-amniotic administration of $80 \mathrm{mg}$ Rivanol (Guangxi Hefeng Pharmaceutical Co., Ltd., Hechi, China). Thirty-six hours later, a fetus with a normal placenta, as well as a partially cystic placenta were delivered (Fig. 2). Suction curettage was performed due to incomplete evacuation. There were no fetal gross abnormalities and no genetic abnormalities were identified by DNA Array (Affymetrix, Inc., Santa Clara, CA, USA). Histopathological analysis of the molar tissue revealed chorionic villi of varying size and shape, with focal edema and functioning villous circulation, as well as focal trophoblastic hyperplasia (Fig. 3). 
A

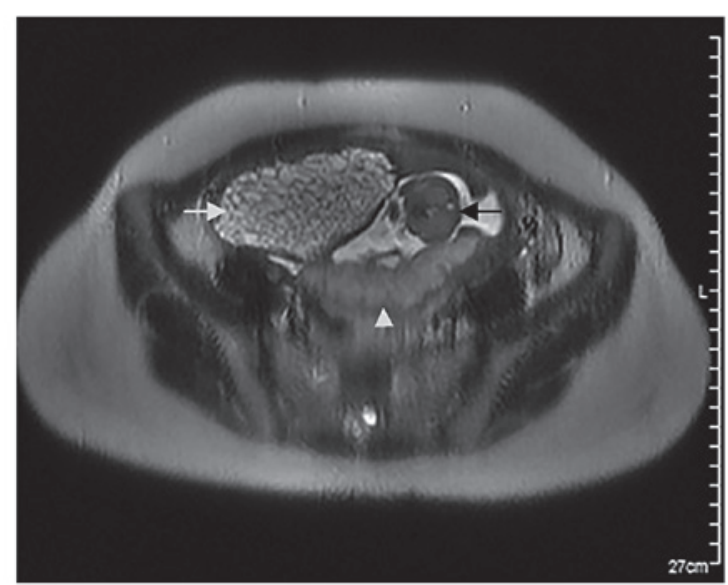

B

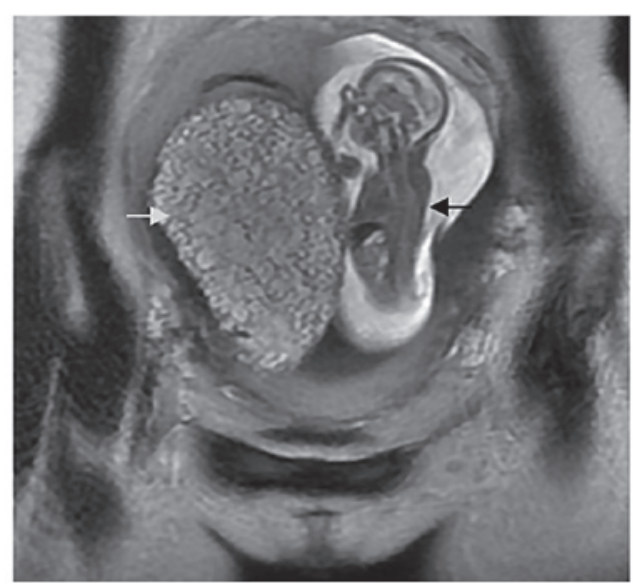

Figure 1. T1-weighted single shot fast spin echo magnetic resonance imaging scans revealing the cystic placenta with a degenerated twin in the right anterior portion of the uterus (white arrow), a normal fetus (black arrow) and placenta in the posterior of the uterus (white arrowhead). (A) Transverse scan and (B) coronal scan.
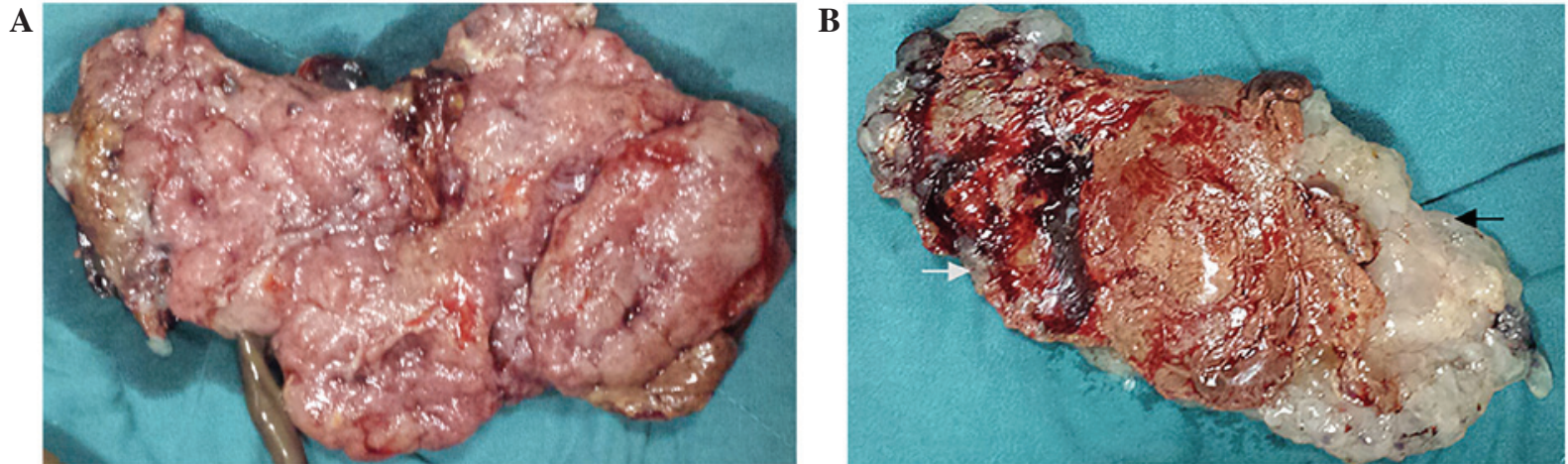

Figure 2. Images of the gross specimen. (A) The normal placenta (specimen size, 12.0x8.0x2.0 $\mathrm{cm}$ ), and (B) the abnormal placenta (specimen size, 11.5x6.0x1.8 cm) containing molar (black arrow) and normal (white arrow) sections.

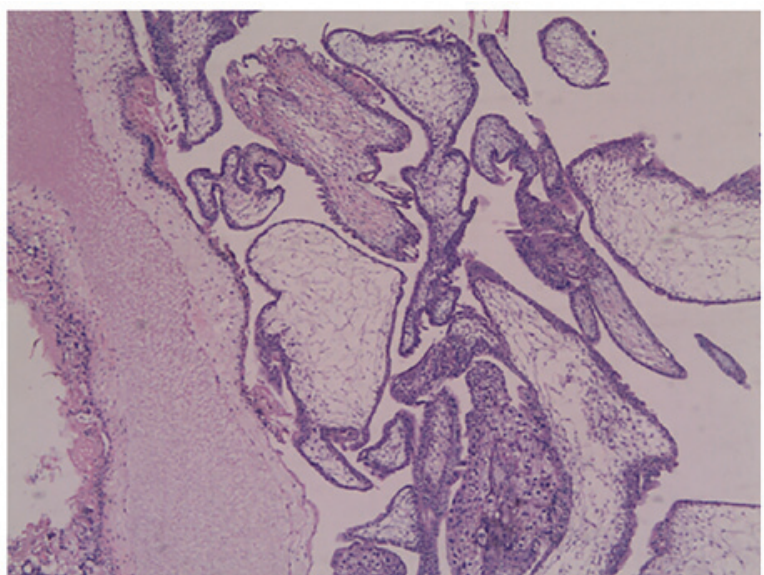

Figure 3. Histopathology of the hydatidiform mole (hematoxylin and eosin staining; magnification, $\mathrm{x} 40$ ) revealed chorionic villi of varying sizes and shapes, with focal edema and functioning villous circulation, as well as focal trophoblastic hyperplasia.

DNA Array (Affymetrix, Inc.) of the molar tissue revealed a triploid cell line, and thus confirmed the diagnosis of PHMCF.

Serum $\beta$-HCG levels were monitored for three weeks following the termination of pregnancy (TOP), and initially reduced to $3,845 \mathrm{IU} / \mathrm{l}$; however, 1 week later, $\beta$-HCG levels had increased to 3,947 IU/l. An MRI study revealed abnormal signs in the right posterior portion of the myometrium and uterine cavity, which indicated the presence of an invasive mole (Fig. 4). Serum $\beta$-HCG levels were observed to have plateaued $(\sim 1,706-2,603 \mathrm{IU} / \mathrm{l})$ at the next two-week follow-up appointment. A follow-up chest computed tomography (CT) scan revealed a number of small, ill-defined nodules of varying sizes in the right and left lower lungs, indicative of lung metastases (Fig. 5). An MRI of the head and a CT scan of the abdomen were normal. The patient was admitted for chemotherapy, and following three courses (3-week cycles) of methotrexate $(0.4 \mathrm{mg} / \mathrm{kg}$, days $1-5)$, the metastases receded and $\beta$-HCG levels reduced to within the normal range. The patient demonstrated no disease recurrence during 1 year of follow-up.

\section{Discussion}

Multiple pregnancy involving a partial hydatidiform mole and normal fetus is rare. The management of such pregnancies presents a dilemma for physicians and parents between expectant management and immediate intervention; particularly in pregnancies involving assisted reproductive technology (8-10). A small number of cases of PHMCF 
Table I. Previously reported cases of partial hydatidiform mole and coexisting fetus in the literature.

\begin{tabular}{|c|c|c|c|c|c|c|}
\hline Study & $\begin{array}{l}\text { Gestational age at delivery } \\
\text { or abortion, weeks }\end{array}$ & Method of termination & Live neonate & PTD & Metastases & Ref \\
\hline Ingec et al & 10 & D\&E & No & Yes & No & (14) \\
\hline Tay & 11 & $\mathrm{D} \& \mathrm{E}$ & No & No & No & $(8)$ \\
\hline Kim et al & 14 & Medical & No & Yes & Yes $^{\mathrm{a}}$ & (9) \\
\hline Zhou et al & 16 & Medical & No & Yes & Yes $^{\mathrm{a}}$ & $(10)$ \\
\hline Shiina et al & 20 & Unknown & No & Yes & Yes $^{\mathrm{a}}$ & $(15)$ \\
\hline Sánchez-Ferrer et al & 21 & Spontaneous abortion & No & No & No & (16) \\
\hline Chu et al & 24 & Caesarean section & Yes & No & No & $(17)$ \\
\hline Copeland and Stanek & 28 & Caesarean section & Yes & No & No & (11) \\
\hline Navarro Amezcua et al & 29 & Caesarean section & No & No & No & (18) \\
\hline Sun et al & 35 & Caesarean section & Yes & No & No & $(12)$ \\
\hline Present case & 17 & Medical & No & Yes & Yes & \\
\hline
\end{tabular}

${ }^{\text {a } m e t a s t a s e s ~ l o c a t e d ~ i n ~ t h e ~ l u n g . ~ P T D, ~ p e r s i s t e n t ~ t r o p h o b l a s t i c ~ d i s e a s e ; ~ D \& E, ~ d i l a t a t i o n ~ a n d ~ e v a c u a t i o n . ~}$

A

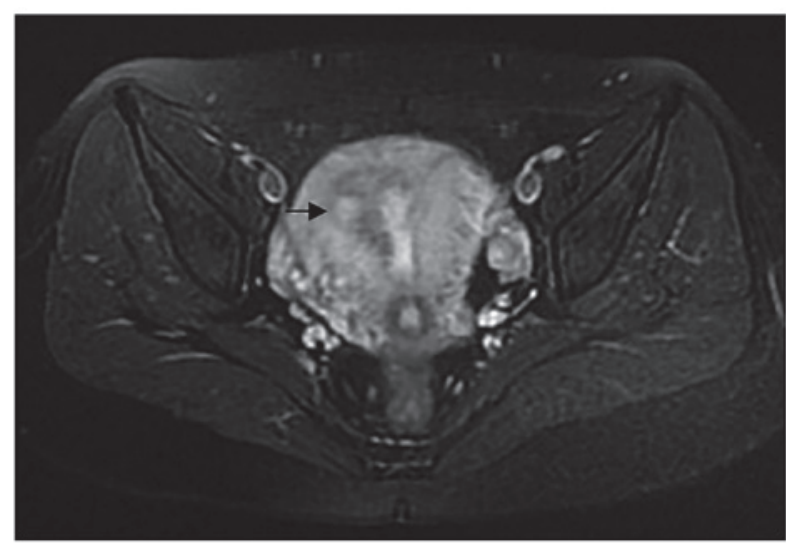

B

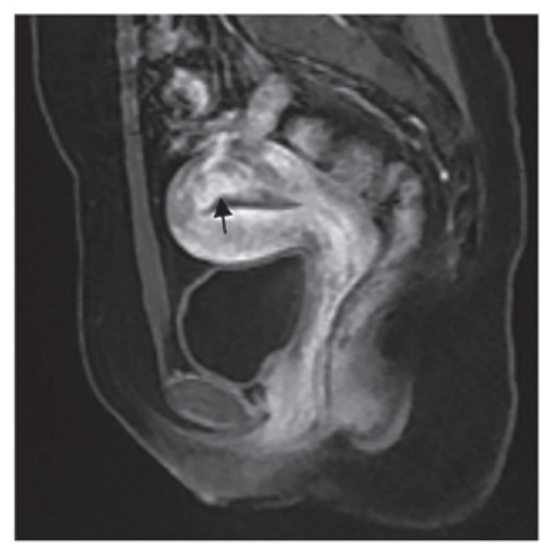

Figure 4. MRI scanning revealed abnormal signs in the right posterior portion of the myometrium and uterine cavity, indicating an invasive mole (A) Transverse T2-weighted fat-suppressed fast recovery fast spin echo MRI, and (B) sagittal LAVA-flex image with contrast enhancement. Arrows indicate the trophoblastic tumor. MRI, magnetic resonance imaging.

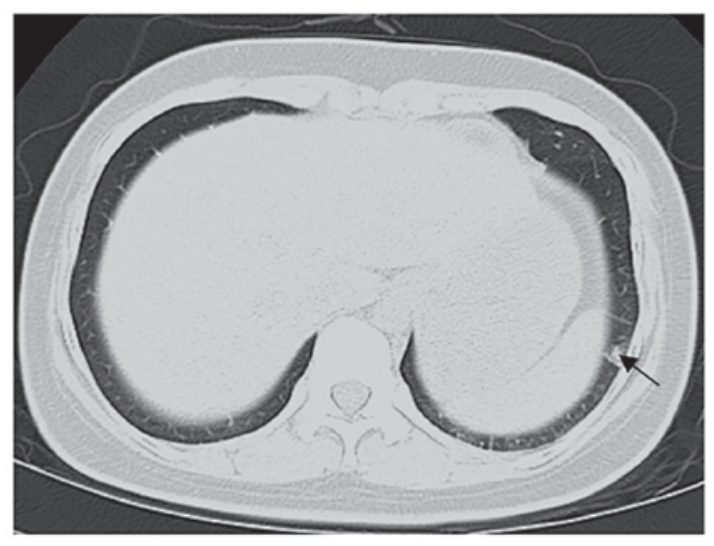

Figure 5. Computed tomography chest scan revealing the lung metastasis of the trophoblast. Arrow indicates the metastatic tumor.

where pregnancy was allowed to continue until 28 weeks of gestation or later have previously been reported, and delivery was achieved via Caesarean section, resulting in a number of healthy babies $(11,12)$. However, parents must also be aware of the risk of potential maternal complications associated with molar pregnancy, including early-onset preeclampsia, hyperemesis gravidarum, PTD and metastases (13).

To the best of our knowledge, following an extensive search of the relevant medical literature since the year 2000, 10 PHMCF cases have previously been reported (Table I) (8-12,14-18). Two of these cases of PHMCF were treated by termination of pregnancy during the first trimester, via dilatation and suction curettage, resulting in one case of PTD and no metastases $(8,14)$. In $3 / 5$ cases $(60 \%)$ that were terminated during the second trimester, two by medically induced labor and the other via unspecified methodology, the patient subsequently developed PTD and lung metastases $(9,10,15)$. Finally, three cases that were terminated by caesarean section in the third trimester did not develop PTD or metastases, and resulted in the delivery of live infants (although one infant succumbed 30 days subsequent to delivery) $(11,12,18)$. In the present case, the PHMCF pregnancy was terminated by the induction of labor via intra-amniotic administration of Rivanol 
at 17 weeks of gestation. PTD and pulmonary metastases were subsequently detected, which required further treatment with chemotherapy. Certain investigators have hypothesized that the risk of PTD in HMCF pregnancies is not associated with the length of gestation at termination (5). The literature cited in the present study also supports this conclusion, as none of the three cases where pregnancy was terminated later than 28 weeks of gestation developed PTD.

Although advances in transvaginal ultrasound and MRI may assist with the diagnosis of HMCF in the first trimester $(8,19)$, HMCF is typically diagnosed at $\sim 15-20$ weeks of gestation or later (20). The empirical management of pregnancy in the second trimester is more difficult (10). Suction curettage is the typical treatment for females exhibiting molar pregnancy, however normal fetal structures may preclude the use of this method (7). Medical induction of labor is an effective and safe way for the termination of normal pregnancies during the second trimester (21). However, medical termination of molar pregnancy is associated with an increased risk of a subsequent requirement for chemotherapeutic treatment, due to the development of increased intrauterine pressure in the first and second trimesters (7). Performance of a caesarean section is not recommended during the second trimester of pregnancy, due to the increased risk of maternal morbidity and the fact that the reduced gestational period means that the neonate is too small to survive (7).

Evidence-based practice guidelines of the Royal College of Obstetricians and Gynecologists recommend dilatation and evacuation (D\&E) as a safe and effective termination option for pregnancies of $>15$ weeks gestation, when undertaken by specialist practitioners with sufficient experience and skills (22). A number of studies have demonstrated that $\mathrm{D} \& \mathrm{E}$ is a rapid and well-tolerated method of termination, demonstrating low complication rates when performed by skilled physicians $(23,24)$. However, to the best of our knowledge, there have been no reports concerning the risk of PTD following PHMCF termination by D\&E. In a study conducted by Niemann et al (5), 5/8 HMCF patients' pregnancies were terminated by curettage during the second trimester. One of these patients developed PTD, indicating a risk factor of $20 \%$.

Mid-trimester TOP due to PHMCF is challenging $(9,10)$. Due to the rarity of this condition, minimal relevant data has been amassed, meaning that it is difficult to formulate valid predictions for treatment and outcomes of patients presenting with PHMCF during the second trimester. The present study concluded that medical termination may not be a safe method for patients with PHMCF, due to the high risk of PTD and metastasis associated. D\&E may be an effective alternative method for termination of PHMCF in the second trimester. Pregnancy may be allowed to continue empirically, provided that maternal morbidity does not appear to be associated with the gestational age at termination, and that the acquisition of healthy neonates is a feasible outcome.

\section{Acknowledgements}

The authors would like to thank Professor Jian-Ming Zhu of the University of North Carolina School of Medicine (Chapel Hill, NC, USA) for his contribution in preparing this manuscript and for his discussion.

\section{References}

1. Jones WB and Lauersen NH: Hydatidiform mole with coexistent fetus. Am J Obstet Gynecol 122: 267-272, 1975.

2. Steller MA, Genest DR, Bernstein MR, et al: Natural history of twin pregnancy with complete hydatidiform mole and coexisting fetus. Obstet Gynecol 83: 35-42, 1994.

3. Sebire NJ, Foskett M, Paradinas FJ, et al: Outcome of twin pregnancies with complete hydatidiform mole and healthy co-twin. Lancet 359: 2165-2166, 2002.

4. Peng M, Li L, Zheng J, Ding Y, Yu L and Huang J: Termination of twin pregnancies with hydatidiform moles: A case series of four patients. Iran J Public Health 43: 1000-1006, 2014.

5. Niemann I, Sunde L and Petersen LK: Evaluation of the risk of persistent trophoblastic disease after twin pregnancy with diploid hydatidiform mole and coexisting normal fetus. Am J Obstet Gynecol 197: 45.e1-45.e5, 2007.

6. Worley MJ Jr, Joseph NT, Berkowitz RS and Goldstein DP: Women with a partial mole during their first pregnancy and diagnosed earlier in gestation are at increased risk of developing gestational trophoblastic neoplasia. Int J Gynecol Cancer 24: 941-945, 2014.

7. Tidy JA, Gillespie AM, Bright N, Radstone CR, Coleman RE and Hancock BW: Gestational trophoblastic disease: A study of mode of evacuation and subsequent need for treatment with chemotherapy. Gynecol Oncol 78: 309-312, 2000.

8. Tay ET: Partial hydatidiform mole and coexisting viable twin pregnancy. Pediatr Emerg Care 29: 1298-1300, 2013.

9. Kim CH, Kim YH, Kim JW, Kim KM, Cho MK, Kim SM, Nam JH and Song TB: Triplet pregnancy with partial hydatidiform mole coexisting with two fetuses: A case report. J Obstet Gynaecol Res 34: 641-644, 2008.

10. Zhou X, Chen Y, Li Y and Duan Z: Partial hydatidiform mole progression into invasive mole with lung metastasis following in vitro fertilization. Oncol Lett 3: 659-661, 2012.

11. Copeland JW and Stanek J: Dizygotic twin pregnancy with a normal fetus and a nodular embryo associated with a partial hydatidiform mole. Pediatr Dev Pathol 13: 476-480, 2010.

12. Sun CJ, Zhao YP, Yu S, Fan L, Wu QQ, Li GH and Zhang WY: Twin pregnancy and partial hydatidiform mole following in vitro fertilization and embryos transfer: A novel case of placental mosaicism. Chin Med J (Engl) 125: 4517-4519, 2012.

13. Vimercati A, de Gennaro AC, Cobuzzi I, Grasso S, Abruzzese M, Fascilla FD, Cormio G and Selvaggi L: Two cases of complete hydatidiform mole and coexistent live fetus. J Prenat Med 7: 1-4, 2013.

14. Ingec M, Borekci B, Altas S and Kadanali S: Twin pregnancy with partial hydatidiform mole and coexistent normal fetus. J Obstet Gynaecol 26: 379-380, 2006.

15. Shiina H, Oka K, Okane M, et al: Coexisting true hermaphroditism and partial hydatidiform mole developing metastatic gestational trophoblastic tumors. A case report. Virchows Arch 441: 514-518, 2002.

16. Sánchez-Ferrer ML, Ferri B, Almansa MT, et al: Partial mole with a diploid fetus: Case study and literature review. Fetal Diagn Ther 25: 354-358, 2009.

17. Chu W, Chapman J, Persons DL and Fan F: Twin pregnancy with partial hydatidiform mole and coexistent fetus. Arch Pathol Lab Med 128: 1305-1306, 2004.

18. Navarro Amezcua ME, Castellanos Reyes J, Cardona González O and Torres Gómez LG: Twin pregnancy with partial hydatidiform mole and alive fetus: Case report. Ginecol Obstet Mex 76: 275-279, 2008 (In Spanish).

19. Herek D and Karabulut N: The role of magnetic resonance imaging in the diagnosis of complete hydatidiform mole in a twin pregnancy. Int J Gynaecol Obstet 123: 77, 2013.

20. Matsui H, Sekiya S, Hando T, Wake N and Tomoda Y: Hydatidiform mole coexistent with a twin live fetus: A national collaborative study in Japan. Hum Reprod 15: 608-611, 2000.

21. Mentula MJ, Niinimäki M, Suhonen S, Hemminki E, Gissler M and Heikinheimo O: Immediate adverse events after second trimester medical termination of pregnancy: Results of a nationwide registry study. Hum Reprod 26: 927-932, 2011.

22. Morrison J: Audit of the care of women requesting induced abortion. J Obstet Gynaecol 23: 521-524, 2003.

23. Panoskaltsis TA: Mid-trimester termination of pregnancy by dilatation and evacuation. J Obstet Gynaecol 21: 280-284, 2001.

24. Kent A: Second trimester termination of pregnancy. Rev Obstet Gynecol 4: 95, 2011. 